\title{
The Comparison of Family Function and its Related Factors in First-Child Infertile Women and Second-Child Infertile Women after 'Two-Child' Policy in China
}

\author{
Tian Qiu" ${ }^{1 \#}$, Zhi Ma ${ }^{2,3 *}$, Yong Zhao ${ }^{4,5,6}$, Wenling Wang7, Huimin Jiang ${ }^{2,3}$, Fengdi Wang ${ }^{2,3}$, \\ Yuelu Chen ${ }^{2,3}$, Ting-Li Han ${ }^{3}$, Yang Yang ${ }^{3,8}$ and Lianlian Wang ${ }^{2,3 *}$
}

${ }^{1}$ Department of Psychiatry, The First Affiliated Hospital of Chongqing Medical University, Chongqing, China

${ }^{2}$ Department of Reproductive Center, The First Affiliated Hospital of Chongqing Medical University, Chongqing, China

${ }^{3}$ State Key Laboratory of Maternal and Fetal Medicine of Chongqing Municipality, Chongqing Medical University, Chongqing, China

${ }^{4}$ School of Public Health and Management, Chongqing Medical University, Chongqing, China

${ }^{5}$ Research Center for Medicine and Social Development, Chongqing Medical University, Chongqing, China

${ }^{6}$ The Innovation Center for Social Risk Governance in Health, Chongqing Medical University, Chongqing, China

${ }^{7}$ Gansu Provincial Maternity and Child-Care Hospital, Lanzhou, Gansu, China

${ }^{8}$ Department of Obstetrics and Gynecology, The First Affiliated Hospital of Chongqing Medical University, Chongqing, China

${ }^{\#}$ Equal Contribution

*Corresponding author: Lianlian Wang, Department of Reproductive Center, The First Affiliated Hospital of Chongqing Medical University, Chongqing 400016, China, Tel: 18908322299

\begin{abstract}
Background: The increasing prevalence of infertility rate around the world has seriously impacted individuals, couples and families. This research identifies how infertility influences family functions in China under the newly introduced two-child policy. Specially, we compared family function between first-child infertile (FI) and second-child infertile (SI) women.

Methods: This study included 572 infertile women of childbearing age, composed with 337 first-child infertile women and 235 second-child infertile women in a reproductive medical center in Chongqing, China, between Feb 2016 to Dec 2018. The score of family perception were measured by Family Assessment Device (FAD) and Family Cohesion and Adaptability Evaluation Scale II (FACES II). The score of depression was measured using the Patient Health Questionnaire-9 (PHQ-9).

Results: The demographic statistics of our sample showed the age of $\mathrm{FI}$ was $33.26 \pm 3.46$, while the SI was $36.68 \pm$ $5.01 .46 \%$ of $\mathrm{FI}$ women are only child, while $23.8 \%$ of SI
\end{abstract}

women are only child $(P<0.001)$. FI women had higher education levels than $\mathrm{SI}$ women $(P<0.001)$. FI women made higher income than $\mathrm{SI}$ did $(P<0.001)$. The psychiatric diagnostics shows $170(50.4 \%)$ of $\mathrm{FI}$ families and 157 $(66.8 \%)$ of SI families had a poor family function $(P<0.001)$. According to the correlation analysis, we found that infertility women with lower education level and lower family income are more associated with an unhealthy family function ( $p$ $<0.05)$. The total score of PHQ-9 is related to the family function $(p<0.05)$.

Conclusions: The socio-demographical statistics show SI women have lower family monthly income and lower education levels in comparison with $\mathrm{FI}$ individuals. Regarding the family functioning, SI women obtained worse scores and more family dysfunction rate. The study indicated that in general, infertile families have higher risks in family functioning, family cohesion and family adaptability. Counselors and psychologists can be deployed in infertility centers to screening people with family problem risks from the beginning and perform early interventions to improve family problems.

Citation: Qiu T, Ma Z, Zhao Y, Wang W, Jiang H, et al. (2022) The Comparison of Family Function and its Related Factors in First-Child Infertile Women and Second-Child Infertile Women after 'Two-Child' Policy in China. Int J Clin Biostat Biom 8:044. doi.org/10.23937/2469-5831/1510044

Accepted: January 07, 2022: Published: January 09, 2022

Copyright: (c) 2022 Qiu T, et al. This is an open-access article distributed under the terms of the Creative Commons Attribution License, which permits unrestricted use, distribution, and reproduction in any medium, provided the original author and source are credited. 


\section{Keywords}

Family functioning, Depression, Family assessment device, Family adaptability and cohesion evaluation scales II, Infertility

\section{Abbreviations}

FI: First-Child Infertility; SI: Second-Child Infertility; OR: Odds Ratio; SD: Standard Deviation; Ref: Reference

\section{Statement of Significance}

After China's "two-child" policy, the family function of infertile families has attracted wide attention. However, no research has investigated the specific family function of infertile families and its possible influence on infertility. The purpose of this study was to compare the differences in family function between first-child infertility and second-child infertility families in China after the two-child policy and their possible influencing factors.

\section{Introduction}

Infertility is a disease characterized by the failure to achieve a clinical pregnancy after 12 months of regular, unprotected sexual intercourse or due to an impairment of a person's capacity to reproduce, either as an individual or with his/her partner [1]. Previous studies have reported that $15.5 \%$ of reproductive-age women in the United States suffer from infertility [2], as well as $24 \%$ in France [3] and 25\% in China [4]. We define that a first-child infertile female is a woman who has never been diagnosed with a clinical pregnancy and meets the criteria of infertility, while second-child infertility applies to a woman unable to establish a clinical pregnancy but who has previously been diagnosed with a clinical pregnancy and had a baby already.

As in many cultures, as a woman, having a child is regarded as a major contribution to family and social life cycle. The inability to reproduce naturally can cause feelings of shame, guilt, and low self-esteem. These negative feelings may lead to varying degrees of depression, anxiety, distress, and a poor quality of life. Therefore, infertility is thought of a major life crisis which causes serious mental problems and stressful experience for those women [5]. For example, high anxiety [6], low marital and sexual satisfaction [7], more psychiatric disorders $[6,8,9]$, reduced quality of life [10], and high depression prevalence $[6,11,12]$ have been reported in infertile patients. Many studies had reported that infertile women are prone to depression and anxiety. Social relationship and family function $[13,14]$ are critical issues related to mental health.

Family functioning refers to the ability of the family to work together as a unit to satisfy the basic needs of its members [15]. More than one million infertile couples living in Iran and infertility is one of the major causes of their divorce [16].
The first-child infertile women are considered to suffer more stress than the second-child infertile women because they have no baby yet. But detailed research comparing their levels family dysfunction is not conducted.

To sum up, infertile women have many problems that may affect family relationships and may lead to many negative consequences including impairment of infertility treatment process and reduction of their mental health. On the other hand, in 2016, the new universal two-child policy was introduced in China enabling all Chinese couples to have a second child. A government-led national survey revealed that most women included under the policy would be 35-years-old and older and thus would be at higher risk of infertility. Although research on family and social relationships of these families has been done, these studies have not compared the different family functions between $\mathrm{FI}$ women and SI women after China's two child policy implementation.

Therefore, this study was designed to compare family functioning between $\mathrm{FI}$ and $\mathrm{SI}$ women and find out the possible factors attribute to their family function after the new policy implementation.

\section{Methods}

\section{Participants}

This study was conducted at the Reproductive Medical Center over a period of 22 months from February 2016 to December 2018.

We used an interview to determine whether the participants met the following criteria (a) Willing to participate; (b) Age over 18 and under 50; (c) Premenopausal; (d) Been unsuccessful in conceiving a baby with unprotected intercourse for more than 1 year; (e) Ability of Chinese reading and writing.

Among all the screened participants, there were 572 infertile women who met the research criteria and fulfilled the questionnaires in the study, among them there were 337 first-child infertile women and 235 second-child infertile women. Social demographic characteristics were evaluated by a demographic questionnaire, designed by the researchers, including gender, age, marriage status, single child or not, educational level, family history of psychosis, family monthly income and fertility attitude and so on.

\section{Measures}

(https://pic3.zhimg.com/v2-7fece433d0b01f2668c8 ae6fdcebdba2_b.jpg)

The General Functioning Scale of the McMaster Clinical Rating Scale [FAD-GFS]: The FAD-GFS provides an overall measure of the health or pathology of the family and was the instrument employed in this study. The FAD-GFS is a 12-item scale comprising 6 
items describing healthy family functioning and 6 items describing unhealthy family functioning. Low scores indicate healthy family functioning. It is highly correlated (range $r=0.48-0.76$ ) with each of the six dimensional scales [17] and is recommended for use as a brief version of the FAD [18]. Based on theory and empirical evidence, Miller, Epstein, Bishop and Keitner [19] recommend a clinical cut-off score of 2.00 on the FAD-GFS to discriminate healthy from unhealthy family functioning. They report a diagnostic confidence rating of 0.83 for the scale [20].

The Family Adaptability and Cohesion Evaluation Scale, second edition, Chinese version (FACESII-CV): FACES II is a 30-item self-report scale that evaluates two dimensions of family life: Family cohesion and family adaptability. Each of the items is scored on a 1-5 Likert format scale with higher score indicating better family functioning. The FACES II has been translated into Chinese (FACESII-CV) in 1992 and shows both a good internal consistency $(0.73 \sim 0.85)$ and test-retest reliability (0.84 0.91). And in the study of Phillips [21], FACESII-CV also shows a good psychometric property. Usually, respondents are asked to answer the questions twice, first to evaluate subjects' perception of actual family cohesion and adaptability second time to evaluate their perception of ideal family conditions. In this study, we measure both the actual family cohesion and adaptability as we wanted to know the characteristics of family functioning among participants. Levels of dissatisfaction with family functioning are measured as follows: Participants answer all 30 items twice. The first time, they rate their perception of actual conditions in the family, and the second time they rate their ideal family condition. The difference between perceptions of actual and ideal conditions yields a discrepancy score, which measures participants' dissatisfaction with family functioning.

FAD-GFS and FACES II evaluate different aspects of family functioning, as they originated from different theory systems. To better understand the characteristics of family functioning among interfile individuals, both FAD-GFS and FACES II are used to assess perceived family functioning of infertile individuals in our study.

\section{The Patient Health Questionnaire-9 (PHQ-9)}

The PHQ-9 is a self-report scale for detecting a Major Depressive Disorder (MDD) [22] based on diagnostic criteria from the Diagnostic and Statistical Manual of Mental Disorders, Fourth Edition (DSM-IV).It refers to the previous 2-week interval and consists of 9 items of depression symptoms plus a question about functional impairment. Each item is scored on a scale from 0 to 3, higher scores indicate greater depression (the total scores range is $0-27$ ). Scores $\geq 10$ indicates possible depression.

\section{Statistical analysis methods}

SPSS 22 software was used for data analysis. The socio-demographic and family functioning features were described using mean values with standard deviations or frequencies with percentages according to the nature of the variable. We examined for normality and homogeneity of various variables. For the comparisons of socio-demographic characteristic, independent Student's t-test was used when normality assumption hold, Pearson chi-square test was employed for categorical variables. Independent student's t-test was also used for the comparisons of family functioning measured by FAD-GFS and FACES-II between FI and SI women. In order to investigate the possible factors of infertile family functioning, binary logistic regression was calculated. In all statistical tests, a value of $p<0.05$ was considered significant.

\section{Results}

\section{Sociodemographic characteristics of $\mathrm{FI}$ and $\mathrm{SI}$ women}

There were 572 participants in the study, among which 337 individuals were first-child (FI) infertile women, 235 were second-child (SI) infertile women. The age of FI was $33.26 \pm 3.46$, while the SI was $36.68 \pm$ 5.01 . $46 \%$ of $\mathrm{FI}$ women are single child, while $23.8 \%$ of SI women are single child in their family $(p<0.001)$. FI women had higher education levels than SI women $(p<$ 0.001 ). FI women made higher family monthly income than SI did $(p<0.001)$.

The time of infertility of $\mathrm{FI}$ was $4.23 \pm 3.29$ years, while the SI was $3.87 \pm 3.41$ years $(p=0.37)$. The detail sociodemographic description of $\mathrm{FI}$ and $\mathrm{SI}$ women were presented in Table 1.

\section{Family functioning in the $\mathrm{FI}$ and $\mathrm{SI}$ groups}

FAD-GFS and FACES II were used to assess perceived family functioning of infertile individuals in this study. Mean scores on the FAD-GFS were $1.96(S D=0.34)$ for FI women and $2.07(S D=0.38)$ for SI women. Using the clinical range cut-off point of 2 , recommended by Miller, et al. [19], 167 (49.6\%) of the FI families scored within the clinical range, while $78(33.2 \%)$ of the SI families scored within the clinical range. 170 (50.4\%) of FI families and 157 (66.8\%) of SI families scored above the cut-off point $(p<0.001)$ (Table 2).

We used both the perceived and ideal dimensions to measure the characteristics of family functioning among participants. On average, there were no significant differences for $\mathrm{FI}$ and $\mathrm{SI}$ women in the dimensions of perceived family cohesion, perceived and ideal family adaptability as reported in Table 2. While SI's report of the ideal family cohesion scale of FACES-II was significantly higher compared to $\mathrm{FI}$ women $(p<0.001$ for the ideal $\mathrm{CO}$ ). The family dissatisfaction cohesion were no significant differences for $\mathrm{FI}$ and $\mathrm{SI}$ women ( $p$ $=0.883$ ), while there were significant differences in the dimensions of ideal family cohesion $(p=0.001)$. 
Table 1: Demographic description of $\mathrm{FI}$ and SI women.

\begin{tabular}{|c|c|c|c|c|}
\hline & $\begin{array}{l}\text { First-child Infertility } \\
(\mathrm{n}=337)\end{array}$ & $\begin{array}{l}\text { Second-child Infertility } \\
(n=235)\end{array}$ & $F / X^{2}$ & $P$ \\
\hline Age (Mean \pm SD) & $33.26 \pm 3.46$ & $36.68 \pm 5.01$ & 36.41 & $<0.001$ \\
\hline Only-child, number (\%) & $155(46 \%)$ & $56(23.8 \%)$ & 29.22 & $<0.001$ \\
\hline Husband only-child number (\%) & $141(41.8 \%)$ & $45(19.1 \%)$ & 32.49 & $<0.001$ \\
\hline Education, number (\%) & & & 39.62 & $<0.001$ \\
\hline$\leq$ High School $(113,19.8 \%)$ & $40(11.9 \%)$ & $73(31.1 \%)$ & & \\
\hline Middle School $(75,13.1 \%)$ & $39(11.6 \%)$ & $36(15.3 \%)$ & & \\
\hline Undergraduate $(353,61.7 \%)$ & $234(69.4 \%)$ & $119(50.6 \%)$ & & \\
\hline Graduate and above $(31,5.4 \%)$ & $24(7.1 \%)$ & $7(3.0 \%)$ & & \\
\hline Family monthly income (RMB), number (\%) & & & 15.85 & $<0.001$ \\
\hline$\leq 4500(192,33.6 \%)$ & $91(27.0 \%)$ & $101(43.0 \%)$ & & \\
\hline $4501-9000(253,44.2 \%)$ & $164(48.7 \%)$ & $89(37.9 \%)$ & & \\
\hline > $9000(127,22.21 \%)$ & $82(24.3 \%)$ & $45(19.1 \%)$ & & \\
\hline
\end{tabular}

Table 2: Comparisons of family functioning and depression between the FI women and the SI women.

\begin{tabular}{|c|c|c|c|c|}
\hline & $\begin{array}{l}\text { First-child Infertility } \\
(\mathrm{n}=337)\end{array}$ & $\begin{array}{l}\text { Second-child Infertility } \\
(n=235)\end{array}$ & $F / x^{2}$ & $P$ \\
\hline PHQ-9 (Mean \pm SD) & $4.09 \pm 3.43$ & $3.40 \pm 3.14$ & 2.32 & 0.13 \\
\hline Mean score of FAD-GFS & $1.96 \pm 0.34$ & $2.07 \pm 0.38$ & 8.33 & 0.004 \\
\hline Family function according FAD-GFS (cutoff $=2$ ) & & & 15.14 & $<0.001$ \\
\hline Healthy family function & $167(49.6 \%)$ & $78(33.2 \%)$ & & \\
\hline Unhealthy family function & $170(50.4 \%)$ & $157(66.8 \%)$ & & \\
\hline \multicolumn{5}{|l|}{ FACES II } \\
\hline \multicolumn{5}{|l|}{ Cohesion } \\
\hline Perceived & $65.57 \pm 9.35$ & $63.38 \pm 8.79$ & 0.65 & 0.419 \\
\hline Ideal & $74.99 \pm 9.89$ & $74.06 \pm 8.46$ & 10.67 & 0.001 \\
\hline Dissatisfaction & $9.43 \pm 5.17$ & $10.68 \pm 5.31$ & 0.02 & 0.883 \\
\hline \multicolumn{5}{|l|}{ Adaptability } \\
\hline Perceived & $43.78 \pm 9.29$ & $40.95 \pm 8.74$ & 2.25 & 0.134 \\
\hline Ideal & $51.92 \pm 6.36$ & $51.96 \pm 6.39$ & 2.34 & 0.127 \\
\hline Dissatisfaction & $8.14 \pm 6.74$ & $11.01 \pm 8.77$ & 24.53 & 0.001 \\
\hline
\end{tabular}

Binary spearman correlation of the possible factors associated with depression of infertile women

Binary spearman correlation was calculated (Table 3 ) to investigate the possible factors associated with depression of infertile women. According to the analysis, infertility women with lower education levels are more likely to have higher mean score of PHQ-9 $(r=-0.108, p=0.01)$.

Infertile women with higher score of FAD-GFS are more likely to have higher mean score of PHQ-9 ( $r=$ -0.128, $p=0.002$ ). Infertile women who experienced more dissatisfaction of family cohesion are easily to have depression $(r=0.094, p=0.024)$.

\section{Binary spearman correlation of the possible factors associated with family function of infertile women}

We used the binary Spearman correlation to investigate the possible factors associated with family function of infertile women (Table 3).

The general functioning of the subscale of FAD was used in our study to investigate the family function of the infertile women. According to the analysis, infertility women with lower education levels and lower family income are more likely to have an unhealthy family function $(p<0.05)$. Meanwhile, infertile women with higher mean score of perceived cohesion and dissatisfaction family cohesion has more unhealthy family function $(p<0.05)$. In this study, we found that when infertile women are the only child, the family function may be better than the non-only child.

On the other hand, we found that the total score of PHQ-9 is related to the family function. As the PHQ9 score increases, the likelihood of suffering from depression increases, and the infertile women are more likely to suffer an unhealthy family function $(p<0.05)$. 
DOI: 10.23937/2469-5831/1510044

ISSN: 2469-5831

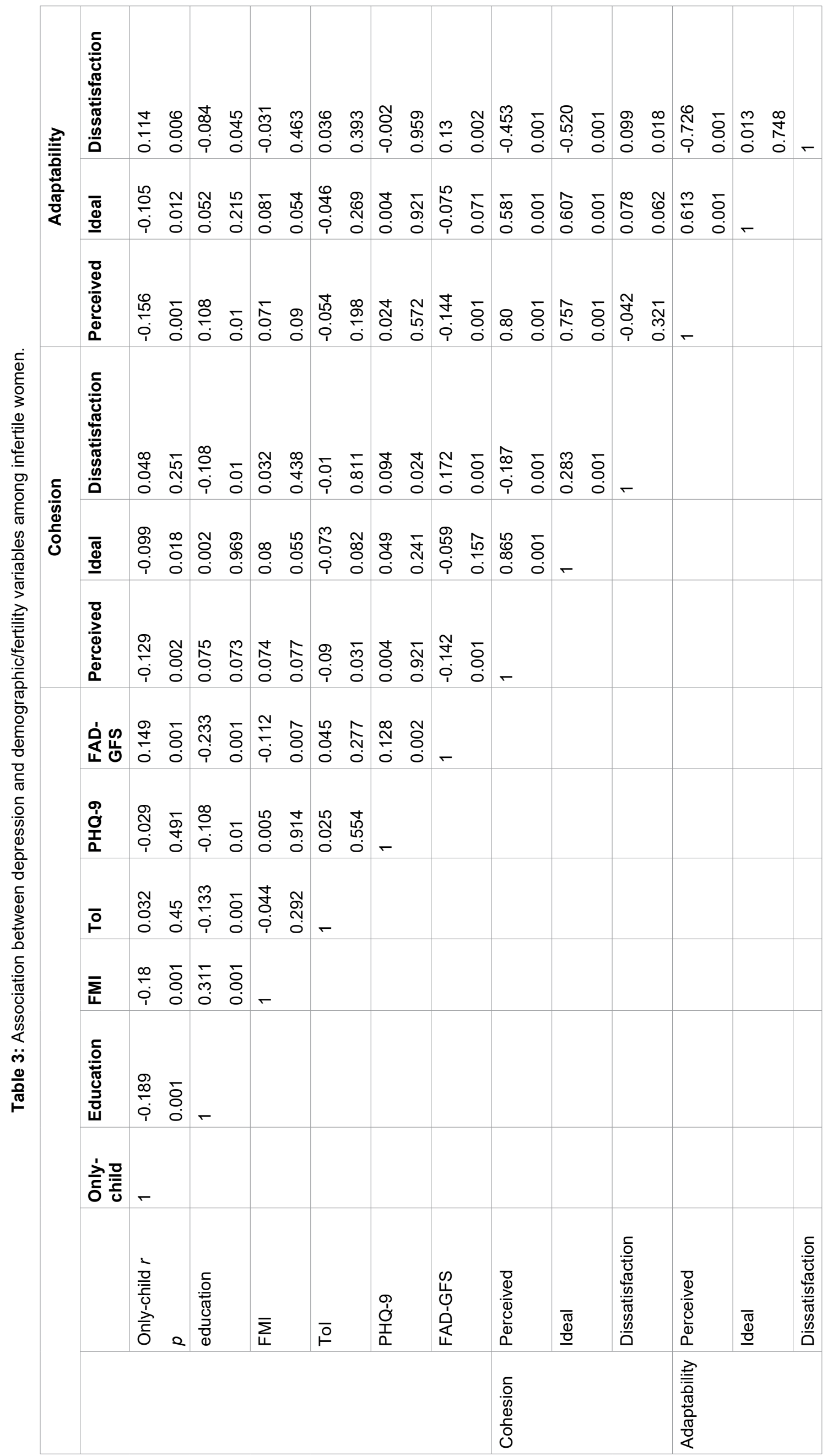

Qiu et al. Int J Clin Biostat Biom 2021, 7:044

- Page 5 of $7 \bullet$ 
According to the correlation analysis of FACES II and the possible factors associated with family functioning, it is found that the more dissatisfaction of family cohesion and adaptability in their family life, the more likely the general family dysfunction is.

\section{Discussion}

In 2016, China introduced the new universal twochild allowing all Chinese couples to have a second child. A government-led national survey revealed that most women included under the policy would be 35-years-old and older and thus would be at higher risk of infertility. This is the first study to use the FAD and FACES II family assessment measures to assess family functioning of infertile female patients in a Chinese infertile woman after the two-child policy implementation. This is also the first study to exploratory the relationship between the family functioning and the mental health of the first-child infertile women and the second-child infertile women in China.

Family function refers to the ability to conduct domestic duties and solve problems as a family member. When facing infertility, a major negative incident, women no surprise feel incapable to solve this crisis: Lack of awareness of the disease, fear of complicated treatment, unsure of treatment outcome and desperation when treatment fails impaired infertile women to low family function. In this study, the results showed that in the subscales of the FACES II, the SI women obtained worse scores, although only in two subscales there was a clear variation among $\mathrm{FI}$ and $\mathrm{SI}$ women. The results also indicated that infertile women have poorer family functioning have many problems in family functioning such as the division of roles, emotional communication, and behavior control [23].

For the social-demographic results in this study, the age of FI women was younger than $\mathrm{SI}$ women, and age was used as covariates when binary logistic regression was performed. Lower family income and lower education levels are more likely attributed to an unhealthy family functioning. Lower family income couldn't provide enough medical insurance for the infertile families, and couples with financial strain are more likely to be related to depression and/or anxiety [24]. Lower education levels are likely impact the communication of the family members. Individuals with low education levels usually find it difficult to exchange information effectively and optimally between family members. All of these will affect family members to collaborate as a unit to satisfy the basic needs of the family members. In this study, it is found that FI has higher family income and higher education level than $\mathrm{SI}$, and the proportion of only children in $\mathrm{Fl}$ is higher than $\mathrm{SI}$, so it is easier for the first infertile women to obtain support from society and their own families. Therefore, we compared the family functions of the two groups. It was found that the general family function of $\mathrm{Fl}$ is better than SI. In the FACES II study comparing FI and SI families, we found that the adaptability in SI families may be poor, which is obviously related to demographic factors such as income level, education level, and poor family functions. Facing infertility, a special disease, patients with better family intimacy and adaptivity are more organized dealing with this crisis, therefore achieve a better family function. Normalization of family function alleviates patients' stress and allows family members positively involve in the treatment.

In this study, the Fl women had higher mean score of PHQ-9 than SI women, and among infertile women, higher mean score of PHQ-9 are a possible risk factor associated to family function. This difference might be reasoned that SI women already have at least a baby. They don't have more fertility related stress internally or their husbands, so the family cohesion and adaptability are more likely better than FI family [25]. What's more, as in Chinese traditional cultures, having a child is considered a major contribution to family and society associated with gender identity. The inability to reproduce naturally can cause guilt, shame, and low self-esteem, all of those may lead to varying degrees of depression, so it is possible that the $\mathrm{FI}$ women have higher risk for depression. Furthermore, individuals with depression usually couldn't perform their duties in the society and family well, and some symptoms of depression were reported to influence the communication and cognitive function of patients. All of these are likely to impact the family functioning.

Generally, it is agreed that physical illnesses have psychological and social aspects. Hence, infertility is considered a bio-psycho-social crisis as well [26]. Especially, after the full liberalization of the 'second child policy', factors such as age, social roles, and intricate family problems all contributed to more complex refertility nature in patients [27]. Thus, the probability of the development of psychological and family disorders in infertile patients is more prevalent than in the other groups, and necessary steps should be taken in this regard.

\section{Discussion}

This study was the initial research of the investigation of family functioning in $\mathrm{FI}$ and $\mathrm{SI}$ families and was evaluated the associated factors related to the family function of fertile individuals. As for social-demographic subscale, the results indicated that SI women have lower family monthly income and lower education levels in comparison with $\mathrm{Fl}$ individuals. Regarding the family functioning aspect, the results showed that the SI women obtained worse scores, and they are more likely have family dysfunction. In general, the result indicated that infertile families have many problems in the general family functioning, family cohesion and family adaptability. 
The results of this research can be used by authorities for future public health and social planning. For example, counselors and psychologists can be deployed in infertility centers to screening people with family problems from the beginning and perform early interventions to improve family problems. Such programs may also have long-term effects such as the mental health of infertile couples and may reduce divorce.

\section{Acknowledgment}

We thank team members for their support and contribution to this study. Especially, thanks for all participants who volunteered to participate in the survey. The authors declare no potential conflicts of interest.

\section{Funding Details}

Chongqing Science and Technology Commission (cstc2018jcyjAX0216);

Joint Program of the Chongqing Science and Technology Bureau and the Chongqing Health Commission (2021MSXM215).

\section{References}

1. Borght MV, Wyns C (2018) Fertility and infertility: Definition and epidemiology. Clin Biochem 62: 2-10.

2. Thoma ME, McLain AC, Louis JF, King RB, Trumble AC, et al. (2013) Prevalence of infertility in the United States as estimated by the current duration approach and a traditional constructed approach. Fertil Steril 99: 1324-1331.

3. Slama R, Hansen OKH, Ducot B, Bohet A, Sorensen D, et al. (2012) Estimation of the frequency of involuntary infertility on a nation-wide basis. Hum Reprod 27: 1489-1498.

4. Zhou Z, Zheng D, Wu H, Li R, Xu S, et al. (2018) Epidemiology of infertility in China: $A$ population-based study. BJOG 125: 432-441.

5. Klemetti R, Raitanen J, Sihvo S, Saarni S, Koponen P (2010) Infertility, mental disorders and well-being--a nationwide survey. Acta Obstet Gynecol Scand 89: 677-682.

6. Chiaffarino F, Baldini MP, Scarduelli C, Bommarito F, Ambrosio $S$, et al. (2011) Prevalence and incidence of depressive and anxious symptoms in couples undergoing assisted reproductive treatment in an Italian infertility department. Eur $\mathrm{J}$ Obstet Gynecol Reprod Biol 158: 235-241.

7. Lee TY, Sun GH, Chao SC (2001) The effect of an infertility diagnosis on the distress, marital and sexual satisfaction between husbands and wives in Taiwan. Hum Reprod 16: 1762-1767.

8. Noorbala AA, Ramezanzadeh F, Abedinia N, Naghizadeh MM (2009) Psychiatric disorders among infertile and fertile women. Soc Psychiatry Psychiatr Epidemiol 44: 587-591.

9. Sbaragli C, Morgante G, Goracci A, Hofkens T, De Leo V, et al. (2008) Infertility and psychiatric morbidity. Fertil Steril 90: 2107-2111.

10. Hassanin IMA, Abd-El-Raheem T, Shahin AY (2010) Primary infertility and health-related quality of life in Upper Egypt. Int J Gynaecol Obstet 110: 118-121.

11. Frederiksen $Y$, O'Toole MS, Mehlsen MY, Hauge B, Elbaek HO, et al. (2017) The effect of expressive writing intervention for infertile couples: A randomized controlled trial. Hum Reprod 32: 391-402.

12. Ramezanzadeh F, Aghssa MM, Abedinia N, Zayeri F, Khanafshar N, et al. (2004) A survey of relationship between anxiety, depression and duration of infertility. BMC Womens Health 4: 9

13. Akyüz A, Sahiner G, Seven M, Bakir B (2014) The effect of marital violence on infertility distress among a sample of Turkish women. Int J Fertil Steril 8: 67-76.

14. Bakhtiyar K, Beiranvand R, Ardalan A, Changaee F, Almasian M, et al. (2019) An investigation of the effects of infertility on Women's quality of life: A case-control study. BMC Womens Health 19: 114.

15. Ryan SR, Friedman CK, Liang Y, Lake SL, Mathias CW, et al. (2016) Family functioning as a mediator of relations between family history of substance use disorder and impulsivity. Addict Disord Their Treat 15: 17-24.

16. Alizadeh Charandabi SM, Kamalifard M, Sedaghiani MM, Montazeri A, Dehghanpour Mohammadian E (2012) Health-related quality of life and its predictive factors among infertile women. J Caring Sci 1: 159-164.

17. Boterhoven de Haan KL, Hafekost J, Lawrence D, Sawyer MG, Zubrick SR (2015) Reliability and validity of a short version of the general functioning subscale of the McMaster Family Assessment Device. Fam Process 54: 116-123.

18. Byles J, Byrne C, Boyle MH, Offord DR (1988) Ontario Child Health Study: Reliability and validity of the general functioning subscale of the McMaster Family Assessment Device. Fam Process 27: 97-104.

19. Miller IW, Epstein NB, Bishop DS, Keitner GI (1985) The McMaster family assessment device. Journal of Marital and Family Therapy 11: 345-356.

20. Wang Y, Yeh YH, Tsang SM, Liu WH, Shi HS, et al. (2013) Social functioning in Chinese college students with and without schizotypal personality traits: An exploratory study of the Chinese version of the First Episode Social Functioning Scale. PLoS One 8: e61115.

21. Phillips MR, West CL, Shen Q, Zheng Y (1998) Comparison of schizophrenic patients' families and normal families in China, using Chinese versions of FACES-II and the Family Environment Scales. Fam Process 37: 95-106.

22. Manea L, Gilbody S, McMillan D (2015) A diagnostic metaanalysis of the Patient Health Questionnaire-9 (PHQ-9) algorithm scoring method as a screen for depression. Gen Hosp Psychiatry 37: 67-75.

23. Zanganeh B, Kaboudi M, Ashtarian H, Kaboudi B (2015) The comparison of family function based on the McMaster model in fertile and infertile women. J Med Life 8: 196-202.

24. Lau JTF, Wang Q, Cheng Y, Kim JH, Yang X, et al. (2008) Infertility-related perceptions and responses and their associations with quality of life among rural chinese infertile couples. J Sex Marital Ther 34: 248-267.

25. Jahromi BN, Mansouri M, Forouhari S, Poordast T, Salehi A (2018) Quality of life and its influencing factors of couples referred to an infertility center in Shiraz, Iran. Int J Fertil Steril 12: 91.

26. Van den Broeck $U$, Emery $M$, Wischmann $T$, Thorn $P$ (2010) Counselling in infertility: Individual, couple and group interventions. Patient Educ Couns 81: 422-428.

27. Lau BHP, Yao SH, Tam MYJ, Chan CLW, Ng EHY, et al. (2019) Gratitude in infertility: A cross-sectional examination of dispositional gratitude in coping with infertility-related stress in women undergoing IVF. Hum Reprod Open 2019: 012. 